\title{
Preoperative Butyrylcholinesterase Level as an Independent Predictor of Overall Survival in Clear Cell Renal Cell Carcinoma Patients Treated with Nephrectomy
}

\author{
Takuya Koie, ${ }^{1}$ Chikara Ohyama, ${ }^{1}$ Jotaro Mikami, ${ }^{1}$ Hiromichi Iwamura, ${ }^{1}$ \\ Naoki Fujita, ${ }^{1}$ Tendo Sato, ${ }^{1}$ Yuta Kojima, ${ }^{1}$ Ken Fukushi, ${ }^{1}$ Hayato Yamamoto,, \\ Atsushi Imai, ${ }^{1}$ Shingo Hatakeyama, ${ }^{1}$ Takahiro Yoneyama, ${ }^{1}$ Yasuhiro Hashimoto, ${ }^{1}$ \\ Masato Kitayama, ${ }^{2}$ and Kazuyoshi Hirota ${ }^{2}$ \\ ${ }^{1}$ Department of Urology, Hirosaki University Graduate School of Medicine, Zaifucho 5, Hirosaki 036-8562, Japan
${ }^{2}$ Department of Anesthesiology, Hirosaki University Graduate School of Medicine, Zaifucho 5, Hirosaki 036-8562, Japan
}

Correspondence should be addressed to Takuya Koie; goodwin@cc.hirosaki-u.ac.jp

Received 18 December 2013; Accepted 29 January 2014; Published 4 March 2014

Academic Editors: Z. Akcetin and A. Tefekli

Copyright (C 2014 Takuya Koie et al. This is an open access article distributed under the Creative Commons Attribution License, which permits unrestricted use, distribution, and reproduction in any medium, provided the original work is properly cited.

\begin{abstract}
The prognostic factors for the overall survival (OS) of clear cell renal cell carcinoma (ccRCC) patients treated with nephrectomy are not well defined. In the present study, we investigated the prognostic significance of preoperative butyrylcholinesterase (BChE) levels in 400 ccRCC patients undergoing radical or partial nephrectomy from 1992 to 2013 at our institution. Univariate and multivariate analyses were performed to determine the clinical factors associated with OS. Among the enrolled patients, 302 were diagnosed with organ-confined disease only (T1-2N0M0), 16 with lymph node metastases, and 56 with distant metastases. The median preoperative BChE level was $250 \mathrm{U} / \mathrm{L}$ (normal range, 168-470 U/L), and median follow-up period was 36 months. The 3year OS rate in patients with preoperative BChE levels of $\geq 100 \mathrm{U} / \mathrm{L}$ was significantly higher than in those with levels of $<100 \mathrm{U} / \mathrm{L}$ (89.3\% versus $77.7 \%, P=0.004)$. On univariate analysis, performance status; anemia; hypoalbuminemia; preoperative levels of $\mathrm{BChE}$, corrected calcium, and C-reactive protein; and distant metastasis status were significantly associated with OS. Multivariate analysis revealed that preoperative BChE levels and distant metastasis status were significantly associated with OS. Our findings suggest a possible role of preoperative BChE levels as an independent predictor of OS after nephrectomy in ccRCC patients.
\end{abstract}

\section{Introduction}

Despite an increase in early diagnosis owing to improved imaging technologies, up to one-third of renal cell carcinoma (RCC) patients are diagnosed with the disease at metastatic stages [1]. Of the remaining two-thirds, approximately $20 \%-40 \%$ of those treated with nephrectomy develop metachronous metastases or local recurrence [2]. Therefore, it is essential to accurately stratify patients according to their overall survival (OS) or recurrence-free survival (RFS) after surgery. Several clinical, pathological, and molecular markers such as C-reactive protein (CRP), preoperative platelet count, and plasma fibrinogen level have enabled more accurate prognosis in RCC [3-6]. However, in general, prognostic factors for OS of RCC patients, particularly those with clear cell RCC (ccRCC), treated with nephrectomy remain poorly defined.

Systemic inflammation is a common host reaction to carcinogenesis or cancer progression [7]. Butyrylcholinesterase (BChE) is an alpha-glycoprotein present in many tissue types, including the central and peripheral nervous system, and in the liver. Low BChE serum levels have been reported in many clinical conditions such as liver damage, inflammation, injury, and malnutrition [8]. A decrease in pretreatment serum BChE level has also been suggested as a useful prognostic parameter in advanced cancer patients with or without hepatic involvement [9-12]. However, the predictive value of serum BChE level in kidney cancer patients is unknown. Thus, in the present study, we aimed to evaluate 
the prognostic significance of pretreatment $\mathrm{BChE}$ levels in patients with ccRCC undergoing nephrectomy.

\section{Materials and Methods}

2.1. Patients. In the present study, we reviewed the clinical charts of 551 patients who underwent partial, radical, or cytoreductive nephrectomy between 1992 and 2013 at a single institution. Our analysis focused on 400 patients who were diagnosed with ccRCC postoperatively. Covariates including age; gender; Eastern Cooperative Oncology Group performance status (PS); preoperative laboratory data on serum levels of hemoglobin, albumin, BChE, corrected calcium (Ca), lactate dehydrogenase (LDH), CRP, and neutrophillymphocyte ratio (NLR); clinical T stage; and distant metastasis status were analyzed. All laboratory data were routinely collected before surgery in all patients. Tumor staging was performed according to the American Joint Committee on Cancer Staging Manual [13]. The study protocol and informed consent documents were reviewed and approved by the Hirosaki University institutional review board.

2.2. Follow-Up Schedule. After nephrectomy, patients with T1 disease received annual followup, whereas those with T2/T3/T4 status were evaluated every 3 months for 2 years and 6 months thereafter. During the follow-up visits, patients underwent physical examination, ultrasonography, and computed tomography (CT).

2.3. Endpoints and Statistical Analysis. The endpoint in this study was overall survival. Data were analyzed using SPSS 22 statistical software (IBM Corp., Armonk, NY, USA). Continuous variables were presented with the median value and interquartile range. Survival after nephrectomy was analyzed using the Kaplan-Meier method. Comparison of subgroup survival was performed using the log-rank test. The covariates examined in the univariate analysis were PS (0 versus $\geq 1$ ), hemoglobin level ( $\geq$ lower limit of normal (LLN) versus $<\mathrm{LLN})$, albumin level $(\geq \mathrm{LLN}$ versus $<\mathrm{LLN})$, BChE level $(\geq 100 \mathrm{U} / \mathrm{L}$ versus $<100 \mathrm{U} / \mathrm{L})$, NLR $(\leq 2.5$ versus $>2.5)$, corrected Ca level ( $\leq 10 \mathrm{mg} / \mathrm{dL}$ versus $>10 \mathrm{mg} / \mathrm{dL}), \mathrm{LDH}(\leq 1.5$ $\times$ upper limit of normal $(\mathrm{ULN})$ versus $>1.5 \times \mathrm{ULN}), \mathrm{CRP}$ ( $\leq 0.3 \mathrm{mg} / \mathrm{dL}$ versus $>0.3 \mathrm{mg} / \mathrm{dL}$ ), T stage (T1 versus T2 versus $\mathrm{T} 3$ versus $\mathrm{T} 4$ ), lymph node status (N0 versus N1), and metastasis status (M0 versus M1). Multivariate analysis was performed using the Cox proportional hazard model. All $P$ values were 2 -sided, and the significance level was set at $<0.05$.

\section{Results}

3.1. Patient Characteristics. The pretreatment characteristics of all patients are listed in Table 1. All diagnoses were conducted based on CT findings. Among the enrolled patients, 302 were diagnosed with organ-confined disease (T1-2N0 M0), whereas 56 had a distant metastasis (any T, any $\mathrm{N}$, and M1). The most frequent metastasis sites were the lungs (36 patients, $64 \%$ ) and the bone (9 patients, $16 \%$ ).

3.2. Oncological Outcomes. The OS was $88.6 \%$ for all 400 enrolled patients (Figure 1). By the end of the follow-up
TABle 1: Patient characteristics.

\begin{tabular}{lc}
\hline Age (years, median, IQR) & $65(56-73)$ \\
Gender $(N, \%)$ & \\
Male & $286(72)$ \\
Female & $114(28)$ \\
Performance status $(N, \%)$ & \\
0 & $368(92)$ \\
$\geq 1$ & $32(8)$ \\
Clinical T $(N, \%)$ & \\
T1 & $261(65)$ \\
T2 & $41(10)$ \\
T3 & $88(22)$ \\
T4 & $10(3)$ \\
Clinical N $(N, \%)$ & \\
N0 & $384(96)$ \\
N1 & $16(4)$ \\
Clinical M $(N, \%)$ & \\
M0 & $344(86)$ \\
M1 & $56(14)$ \\
Followup (months, median, IQR) & $36(17-80)$ \\
\hline IQR: interquatile range $N$ : number; TNM: tumor-node-metastasis staging
\end{tabular}

IQR: interquartile range; $N$ : number; TNM: tumor-node-metastasis staging.

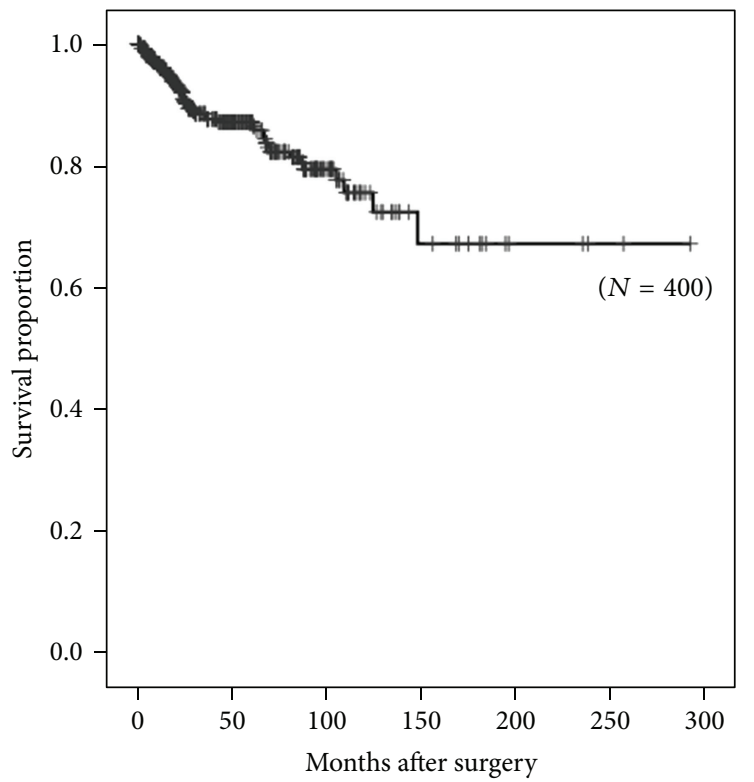

FIGURE 1: Kaplan-Meier estimate of overall survival. The 3-year overall survival rate was $88.6 \%$.

period, 38 patients (10\%) had died of RCC, and 17 (4\%) had died of other causes. Nineteen patients (5\%) remained alive despite metastases from ccRCC.

On univariate analysis, the statistically significant prognostic factors were PS $(P=0.003)$, hemoglobin level $(P=$ 0.004), albumin level $(P=0.010)$, BChE level $(P<0.001)$, corrected Ca level $(P=0.007)$, CRP level $(P<0.001)$, clinical T stage $(P<0.001)$, and metastasis status $(P<$ 0.001 ), as shown in Table 2. Kaplan-Meier curves for OS of all patients, stratified by different serum BChE levels, are shown in Figure 2. 
TABLE 2: Relationship between clinical factors and overall survival in clear cell renal cell carcinoma.

\begin{tabular}{|c|c|c|c|c|}
\hline Variable & Number of patients & Three-year overall survival rate (\%) & 95\% CI & $P$ value \\
\hline \multicolumn{5}{|c|}{ Performance status } \\
\hline 0 & 368 & 89.9 & $198.6-248.9$ & \multirow{2}{*}{0.003} \\
\hline$\geq 1$ & 32 & 72.9 & 77.5-151.1 & \\
\hline \multicolumn{5}{|l|}{ Gender } \\
\hline Male & 286 & 88.4 & $157.3-196.9$ & \multirow{2}{*}{0.596} \\
\hline Female & 114 & 85.8 & $217.2-267.1$ & \\
\hline \multicolumn{5}{|l|}{ Hemoglobin } \\
\hline$\geq \mathrm{LLN}$ & 329 & 92.1 & $191.8-250.9$ & \multirow{2}{*}{0.004} \\
\hline$<\mathrm{LLN}$ & 71 & 72.9 & $152-215$ & \\
\hline \multicolumn{5}{|l|}{ Albumin } \\
\hline$\geq \mathrm{LLN}$ & 282 & 90.3 & $188-254.1$ & \multirow{2}{*}{0.010} \\
\hline$<\mathrm{LLN}$ & 118 & 81.5 & $153.2-206.8$ & \\
\hline \multicolumn{5}{|l|}{ BChE } \\
\hline$\geq 100 \mathrm{U} / \mathrm{L}$ & 356 & 89.3 & 194.6-251.6 & \multirow{2}{*}{0.004} \\
\hline$<100 \mathrm{U} / \mathrm{L}$ & 44 & 77.7 & $101.6-154.8$ & \\
\hline \multicolumn{5}{|l|}{ NLR } \\
\hline$\leq 2.5$ & 287 & 90.2 & $187.5-244.0$ & \multirow{2}{*}{0.457} \\
\hline$>2.5$ & 113 & 81.6 & $114.3-174.1$ & \\
\hline \multicolumn{5}{|c|}{ Corrected calcium } \\
\hline$\leq 10 \mathrm{mg} / \mathrm{dL}$ & 383 & 89.0 & $195.8-245.5$ & \multirow{2}{*}{0.007} \\
\hline$>10 \mathrm{mg} / \mathrm{dL}$ & 17 & 64.5 & $65.5-155.8$ & \\
\hline \multicolumn{5}{|l|}{$\mathrm{LDH}$} \\
\hline$\leq 1.5 \times \mathrm{ULN}$ & 396 & 88.0 & 196.3-241.9 & \multirow{2}{*}{0.551} \\
\hline$>1.5 \times \mathrm{ULN}$ & 4 & 75.0 & $42.5-86.7$ & \\
\hline \multicolumn{5}{|c|}{ C-reactive protein } \\
\hline$\leq 0.3 \mathrm{mg} / \mathrm{dL}$ & 281 & 93.0 & $219.4-265.6$ & \multirow{2}{*}{$<0.001$} \\
\hline$>0.3 \mathrm{mg} / \mathrm{dL}$ & 119 & 76.4 & $116.8-175.6$ & \\
\hline \multicolumn{5}{|l|}{ T stage } \\
\hline $\mathrm{T} 1$ & 261 & 93.0 & $233.8-274.3$ & \multirow{4}{*}{$<0.001$} \\
\hline $\mathrm{T} 2$ & 41 & 88.6 & $101.1-22.6$ & \\
\hline T3 & 88 & 74.4 & $126.1-181.3$ & \\
\hline $\mathrm{T} 4$ & 10 & 77.1 & $36.9-71.6$ & \\
\hline \multicolumn{5}{|l|}{$\mathrm{N}$ stage } \\
\hline N0 & 386 & 89.4 & $198.4-244.9$ & \multirow{2}{*}{0.001} \\
\hline N1 & 14 & 45.3 & $36.7-100.7$ & \\
\hline \multicolumn{5}{|l|}{ M stage } \\
\hline M0 & 344 & 93.4 & $187.3-232.1$ & \multirow{2}{*}{$<0.001$} \\
\hline M1 & 56 & 57.9 & $82.1-167.5$ & \\
\hline
\end{tabular}

CI: confidence interval; BChE: butyrylcholinesterase; LDH: lactate dehydrogenase; NLR: neutrophil-lymphocyte ratio; LLN: lower limit of laboratory's normal range; ULN: upper limit of laboratory's normal range; TNM: tumor-node-metastasis staging.

Multivariate analysis using the Cox proportional hazard model revealed that metastasis status and serum BChE level were independent prognostic predictors for OS (Table 3). No significant relation was noted between the $\mathrm{BChE}$ level and distant metastasis status.

\section{Discussion}

Results from the present study validate the possible role of preoperative serum BChE levels as an independent prognostic factor for OS after surgery in ccRCC patients. To our knowledge, this retrospective analysis is the first report to evaluate the prognostic significance of pretreatment serum BChE levels in ccRCC patients.

Cholinesterases are a group of enzymes that hydrolyze acetylcholine and other choline esters. There are 2 main types of cholinesterases with different biochemical properties [14]. Acetylcholinesterase is present in all excitable tissues-such as the central and peripheral nerve systems and musclesand erythrocytes. The other cholinesterase, $\mathrm{BChE}$, is also present in the nervous system as well as the liver. As BChE is synthesized in the liver, a hepatocellular impairment would 
TABLE 3: Multivariate analysis in 400 patients with clear cell renal cell carcinoma.

\begin{tabular}{lccc}
\hline Variable & Wald & $95 \%$ CI & $P$ value \\
\hline M stage & 27.784 & $0.085-0.324$ & $<0.001$ \\
BChE & 9.022 & $0.173-0.691$ & 0.003 \\
Age & 2.444 & $0.963-1.004$ & 0.118 \\
Hemoglobin & 1.979 & $0.287-1.228$ & 0.160 \\
N stage & 1.226 & $0.268-0.562$ & 0.268 \\
T stage & 0.846 & $0.413-4.179$ & 0.846 \\
C-reactive protein & 0.352 & $0.402-1.629$ & 0.553 \\
Performance status & 0.095 & $0.353-2.135$ & 0.759 \\
Corrected calcium & 0.004 & $0.340-2.752$ & 0.950 \\
Albumin & 0.003 & $0.501-2.081$ & 0.954 \\
\hline
\end{tabular}

CI: confidence interval; BChE: butyrylcholinesterase; TNM: tumor-nodemetastasis staging.

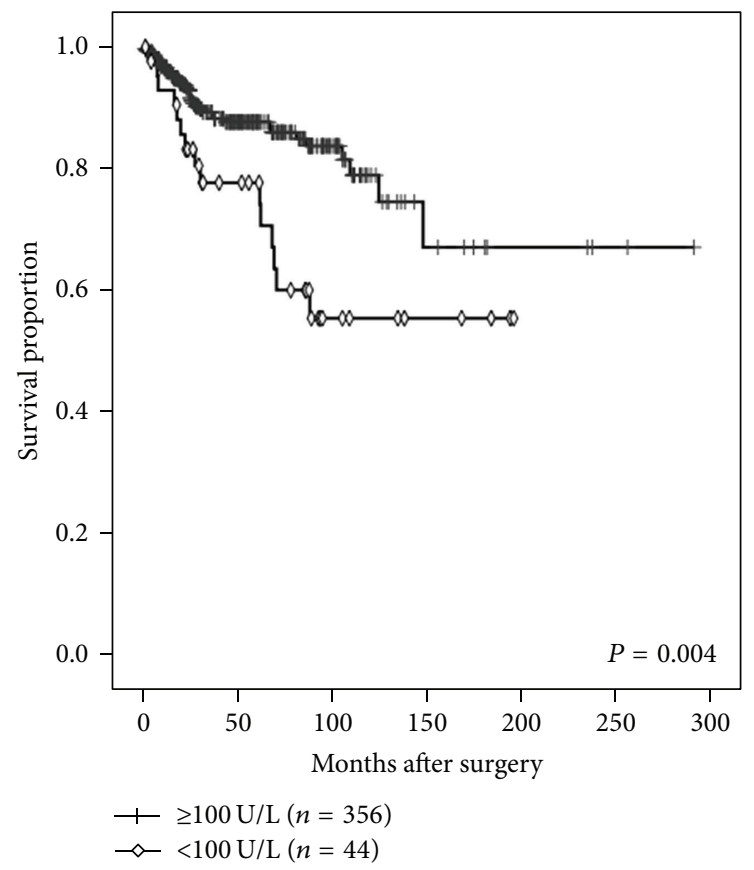

FIGURE 2: Kaplan-Meier estimate of overall survival (OS) according to serum butyrylcholinesterase (BChE) levels. The 3-year OS rate was $89.3 \%$ in patients with preoperative $\mathrm{BChE}$ levels of $\geq 100 \mathrm{U} / \mathrm{L}$ and $77.7 \%$ in those with preoperative BChE levels of $<100 \mathrm{U} / \mathrm{L}(P=$ $0.004)$.

result in a decreased activity of the enzyme. In fact, reduction of BChE levels often occurs in acute and chronic liver damage, cirrhosis, and liver metastasis. Similarly, low BChE levels have been reported during stress and inflammation, as well as in cases of protein-energy malnutrition and other clinical conditions [8].

In geriatric patients, $\mathrm{BChE}$ levels have been suggested as a useful biomarker for malnutrition or a disease prognostic indicator [15]. Levels of CRP, interleukin 6 (IL-6), and tumor necrosis factor alpha (TNF-alpha) have been reported to significantly increase in frail elderly patients, whereas $\mathrm{BChE}$ activity has been observed to significantly decrease $(P<$
0.005) [14]. In addition, BChE activity has been reported to negatively correlate with IL-6 and TNF-alpha levels [14].

Advanced cancer is a clinical condition involving mild to moderate inflammation. Systemic inflammation has been described to be associated with poor prognosis in a variety of malignancies [16]. Plasma BChE levels have been shown to decrease in advanced cancer patients with or without hepatic involvement, despite the other liver function tests yielding normal results [17]. One of the possible mechanisms for such $\mathrm{BChE}$ activity decrease in cancer patients could be secondary anorexia accompanying malignancy [18]. Santarpia et al. suggested that besides albumin levels and Karnofsky index, serum BChE levels were a survival predictive factor in terminal cancer patients with peritoneal carcinomatosis [9]. In another study involving patients with head and neck or uterine cervical cancer, BChE activity was demonstrated to be an effective prognostic marker [10].

The present study has limitations inherent to any retrospective analysis with a limited number of patients. Although $76 \%$ of the enrolled patients were diagnosed with organconfined disease, the other $24 \%$ had an advanced disease with metastases to the lymph nodes or other distant sites. BChE is a sensitive yet nonspecific serum biomarker. Therefore, a decreased BChE level might result from an inflammation or physical stress. In addition, the correlation between the decrease of serum $\mathrm{BChE}$ level and advanced disease or distant metastasis was obscure in this study. However, in ccRCC patients, low serum BChE levels could suggest other systemic disorders, including poor PS or secondary malnutrition. In addition, $\mathrm{BChE}$ levels seem to correlate with cancer activity and nutritional status in ccRCC patients.

\section{Conclusion}

Our results validate the possible role of preoperative serum $\mathrm{BChE}$ levels as an independent prognostic factor after surgery in ccRCC. BChE levels may correlate with cancer activity and nutritional status in ccRCC patients. Therefore, our findings suggest that serum $\mathrm{BChE}$ assessment be included in the routine clinical evaluation of patients with ccRCC.

\section{Conflict of Interests}

The authors declare that there is no conflict of interests regarding the publication of this paper.

\section{Acknowledgment}

The authors thank Ms. Yuki Fujita for her invaluable help with the original data.

\section{References}

[1] J. M. Kozlowski, "Management of distant solitary recurrence in the patient with renal cancer: contralateral kidney and other sites," Urologic Clinics of North America, vol. 21, no. 4, pp. 601624, 1994.

[2] R. J. Motzer, N. H. Bander, and D. M. Nanus, "Medical progress: Renal-cell carcinoma," The New England Journal of Medicine, vol. 335, no. 12, pp. 865-875, 1996. 
[3] M. de Martino, T. klatte, C. Seemann et al., "Validation of serum C-reactive protein (CRP) as an independent prognostic factor for disease-free survival in patients with localised renal cell carcinoma (RCC)," BJU International, vol. 111, no. 8, pp. E348E353, 2013.

[4] S. Steffens, A. Köhler, R. Rudolph et al., "Validation of CRP as prognostic marker for renal cell carcinoma in a large series of patients," BMC Cancer, vol. 12, pp. 399-406, 2012.

[5] S. Brookman-May, M. May, V. Ficarra et al., "Does preoperative platelet count and thrombocytosis play a prognostic role in patients undergoing nephrectomy for renal cell carcinoma? Results of a comprehensive retrospective series," World Journal of Urology, vol. 31, no. 5, pp. 1309-1316, 2013.

[6] J. Du, J. Zheng, X. Chen et al., "High preoperative plasma fibrinogen is an independent predictor of distant metastasis and poor prognosis in renal cell carcinoma," International Journal of Clinical Oncology, vol. 18, no. 3, pp. 517-523, 2013.

[7] M. Trikha, R. Corringham, B. Klein, and J.-F. Rossi, “Targeted anti-interleukin-6 monoclonal antibody therapy for Cancer: a review of the rationale and clinical evidence," Clinical Cancer Research, vol. 9, no. 13, pp. 4653-4665, 2003.

[8] N. Lampón, E. F. Hermida-Cadahia, A. Riveiro, and J. C. Tutor, "Association between butyrylcholinesterase activity and lowgrade systemic inflammation," Annals of Hepatology, vol. 11, no. 3, pp. 356-363, 2012.

[9] L. Santarpia, L. Alfonsi, F. Pasanisi, C. De Caprio, L. Scalfi, and F. Contaldo, "Predictive factors of survival in patients with peritoneal carcinomatosis on home parenteral nutrition," Nutrition, vol. 22, no. 4, pp. 355-360, 2006.

[10] A. Chougule, S. Hussain, and D. Agarwal, "Prognostic and diagnostic value of serum pseudocholinesterase, serum aspartate transaminase, and serum alinine transaminase in malignancies treated by radiotherapy," Journal of Cancer Research and Therapeutics, vol. 4, no. 1, pp. 21-25, 2008.

[11] F. Bozzetti, P. Boracchi, A. Costa et al., "Relationship between nutritional status and tumor growth in humans," Tumori, vol. 81, no. 1, pp. 1-6, 1995.

[12] S. Mitsunaga, T. Kinoshita, T. Hasebe et al., "Low serum level of cholinesterase at recurrence of pancreatic cancer is a poor prognostic factor and relates to systemic disorder and nerve plexus invasion," Pancreas, vol. 36, no. 3, pp. 241-248, 2008.

[13] "Urinary Bladder," in AJCC Cancer Staging Manual, S. B. Edge, D. R. Byrd, C. C. Compton et al., Eds., pp. 497-505, Springer, New York, NY, USA, 7th edition, 2010.

[14] L. Santarpia, I. Grandone, F. Contaldo, and F. Pasanisi, "Butyrylcholinesterase as a prognostic marker: a review of the literature," Journal of Cachexia, Sarcopenia and Muscle, vol. 4, no. 1, pp. 3139, 2013.

[15] C. Mitrache, J. R. Passweg, J. Libura et al., "Anemia: an indicator for malnutrition in the elderly," Annals of Hematology, vol. 80, no. 5, pp. 295-298, 2001.

[16] H. Eggers, C. Seidel, A. J. Schrader et al., "Serum C-reactive protein: a prognostic factor in metastatic urothelial cancer of the bladder," Medical Oncology, vol. 30, no. 4, pp. 705-711, 2013.

[17] S.-Z. Gu, X.-H. Zhao, Q. Ping, S.-B. Li, and B.-R. Pan, "Alterations of serum cholinesterase in patients with gastric cancer," World Journal of Gastroenterology, vol. 11, no. 29, pp. 46044606, 2005.

[18] O. O. Ogunkeye and A. I. Roluga, "Serum cholinesterase activity helps to distinguish between liver disease and non-liver disease aberration in liver function tests," Pathophysiology, vol. 13, no. 2, pp. 91-93, 2006. 